Reprod. Nutr. Dévelop., 1980, 20 (3 B), 843-858.

\title{
Les stimulations olfactives dans les relations entre l'enfant et la mère
}

par B. SCHAAL, (1) H. MONTAGNER, E. HERTLING, D. BOLZONI, A. MOYSE, R. QUICHON *

\author{
Laboratoire de Psychophysiologie, \\ Foculté des Sciences et des Techniques, \\ Route de Gray, 25030 Besançon \\ * Clinique Bon Secours, \\ 18, quai Bugnet, 25000 Besançon.
}

Summary. Olfactory stimulations in mother-child relations.

In this paper, we report new data on mutual olfactory recognition between mother and child.

Newborn infants were filmed since birth. When awakening in the mother's room at the Clinical Hospital, they were confronted with a cotton pad impregnated with diverse secretions of the mother and a cotton pad impregnated with the same secretions of another mother or a pad with no specific odour. We used a double-blind technique. By analyzing the films, frame by frame, we found that the whole area swept by the nose and the arms was significantly decreased in the child whose nose came into contact with the «mother» pad. The method could be used to study other sensory abilities in the baby.

The olfactory abilities of the mother in relation to the baby were also fested with the double-blind principle. We found (i) on the 3rd and 4th days after birth, most mothers recognized the baby's odour, (ii) this faculty decreased from the 4th to the 6th day, and finally (iii) increased from the 8th day. These results are discussed.

We also found that one-third of 3 to 5 years old children more often chose a tee-shirt worn against the mother's skin for 2 to 3 days than any other tee-shirt (worn by an alien mother or with no specific odour).

It appears that olfactory cues can play a role in the attachment of the baby to its mother, and that they still are involved in that process when the child is between 3 and 5 years old.

\section{Introduction.}

Les premiers échanges d'informations du nouveau-né avec son environnement social, en particulier avec sa mère, se font par des voies et des mécanismes encore mal connus et difficiles à explorer par la méthode expérimentale. Pourtant, tout montre que

(1) Pour les demandes de tirages à part, s'adresser à Benoist Schaal. 
ces échanges précoces gouvernent non seulement les inferactions entre le bébé et sa mère, mais influencent aussi l'ensemble du développement sensori-moteur de l'enfant.

Notre groupe de recherche, qui étudie la genèse des systèmes de communication de l'enfant, s'est donc proposé de mieux comprendre les bases sensorielles des échanges d'informations entre l'enfant ef son environnement social, à partir de la naissance. Les études ont porté en particulier sur les informations olfactives. Nous avons d'abord montré que les enfants de 2 à 3 ans peuvent, dans 70 à 75 p. 100 des cas, reconnaître de façon significative un « tee-shirt » qui a été porté par la mère à même la peau pendant 2 à 3 jours (Montagner, 1974a, 1978 ; Montagner et al., 1974b).

La remise du « tee-shirt » imprégné de l'odeur maternelle à des enfants de 2 à 3 ans peut entraîner des modifications de leur comportement, lorsqu'ils sont en activités libres dans la salle de jeux d'une crèche. Certains ont alors tendance à se couper des autres enfants (ils se frottent le visage avec le tricot, le mettent dans leur bouche ou le lèchent...) ; leurs comportements d'agressions se ritualisent et sont moins fréquents qu'avant la remise du tricot ou que les autres jours. Globalement, le nombre de leurs interactions avec les autres enfants diminue par rapport à celui observé dans les moments précédant la remise du tricot et par rapport à la remise de tout autre tricot.

Utilisant un protocole expérimental comparable, Hold et Schleidt (1977) ont montré qu'un tiers des personnes adultes reconnaissent leur propre odeur ou celle du conjoint.

Les recherches sur les « capacités 》 de discrimination olfactive du nouveau-né sont beaucoup plus rares. Cependant, Engen et al. (1963) et Steiner (1973) ont montré que les réponses de nouveau-nés varient selon les odeurs artificielles qu'on leur présente. Dès le premier jour de vie, ils répondent par un retrait de la tête ou une expression faciale caractéristique à la présentation d'odeurs déplaisantes pour l'adulte. Par ailleurs, Mac Farlane (1975) montre que des bébés de 6 jours tournent plus souvent la tête vers un tampon de gaze imprégné de l'odeur du sein de la mère que vers un tampon qui véhicule l'odeur du sein d'une autre mère.

Dans cet article, nous confirmons et précisons cette discrimination olfactive précoce entre une odeur maternelle et une odeur de mère étrangère au moyen d'indices comportementaux différents de ceux de Mac Farlane. Nous rapportons également les premiers résultats que nous avons obtenus sur la reconnaissance olfactive du bébé par la mère.

En même temps que nous avons remonté l'échelle des âges pour étudier la genèse des discriminations olfactives spécifiques du jeune enfant, nous l'avons descendu audelà de 3 ans : nous rapportons donc aussi les données globales que nous avons obtenues dans ce domaine avec des enfants de 3 à 5 ans.

\section{Matériel et méthodes}

1. - La discrimination des odeurs spécifiques entre l'enfant ef sa mère au cours de la période post-natale.

A. - Remarques générales.

C'est au moment des cours d'accouchement sans douleur, quelques semaines avant la naissance, que nous avons sollicité la participation des futurs parents à nos 
expériences. Nous avons ainsi constitué un échantillon global de 20 mères dont les caractéristiques socio-professionnelles sont hétérogènes. Les études ont porté sur ces 20 mères et leur enfant, de la naissance au $10^{\mathrm{e}}$ et parfois $12^{\mathrm{e}}$ jour après la naissance. Les 20 enfants ( 10 garçons et 10 filles) sont cliniquement normaux (score d'APGAR : $10 / 10$ à 1 et à $5 \mathrm{~min}$ ) ; leur âge gestationnel varie entre 38 et 42 semaines.

B. - Tests de discrimination d'odeurs maternelles par l'enfant.

1) Le recueil des odeurs corporelles. - II se fait par application de carrés de gaze $(10 \times 10 \mathrm{~cm})$ sur diverses parties du corps de la mère :

a) le sein : on recueille ainsi le complexe odorant constitué par le mélange des sécrétions lactées et des sécrétions sudoripares et sébacées du sein et du mamelon. Nous demandons aux mères de ne pas s'enduire les mamelons de crèmes protectrices avant ou pendant l'application des tampons de gaze. La durée d'application des tampons varie de 12 à 24 h selon les cas ;

b) le cou et le creux de l'épaule, zones corporelles vers lesquelles les mères orientent souvent la tête de leur bébé : on recueille ainsi un complexe d'odeurs naturelles (glandes sudoripares et sébacées) et d'odeurs artificielles (parfum, eau de toilette...). Les résultats des tests réalisés avec ces odeurs sont exposés par ailleurs (Schaal ef al., à paraître).

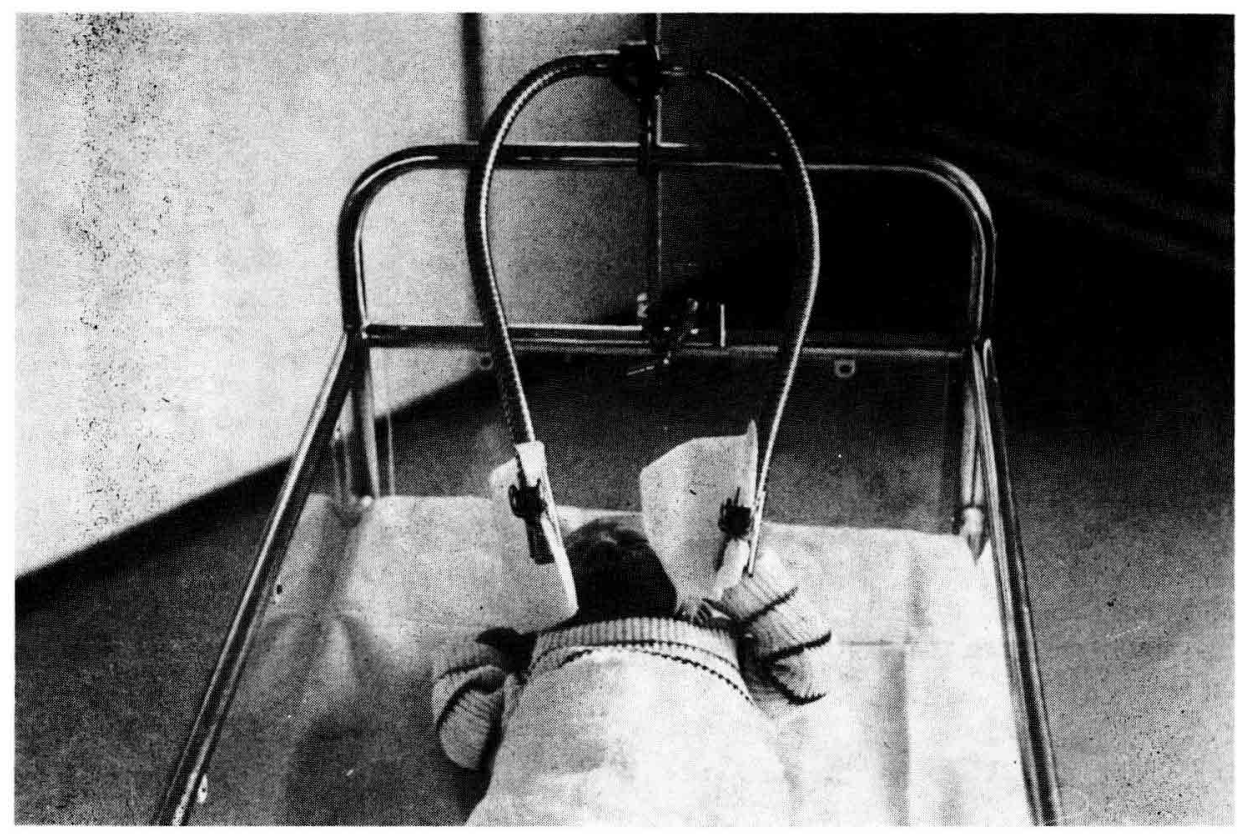

FIG. 1. - Le dispositif expérimental qui permet d'étudier le comportement du bébé dont le nez entre en contact avec un tampon de gaze imprégné des sécrétions maternelles ou un tampon de gaze imprégné des mêmes sécrétions d'une autre mère, ou un tampon olfactivement "neutre " (non imprégné d'une odeur spécifique). Pour plus de détails, voir le texte. 
2) Déroulement des tests.

- Les bébés sont tous testés au même moment de la journée (entre 9 et $10 \mathrm{~h}$ ), avant une tétée. Ils sont alors à des stades de vigilance équivalents : stades IV ou $\mathrm{V}$ de Prechtl (Prechtl, 1965 ; Hutt et al., 1969).

- Les tests se font dans la chambre où séjourne la mère. La chambre est silencieuse. La température ambiante est constante. L'intensité de la lumière du jour est la même de part et d'autre du visage de l'enfant.

- Le bébé est couché sur le dos dans son berceau.

- Un expérimentateur $A$ recueille les 2 tampons de gaze portés par la mère et en fixe un à l'une des branches latérales du dispositif expérimental ; l'autre branche latérale reçoit, selon l'expérience, soit un tampon vierge de toute odeur spécifique, soit un tampon imprégné des sécrétions d'une autre mère (fig. 1).

- Un expérimentateur $B$ tourne la tête du bébé de $45^{\circ}$ de chaque côté de la ligne sagittale de façon que le nez entre en contact successivement avec les 2 tampons de gaze ; puis, la tête est replacée selon le plan de symétrie du corps. L'expérimentateur $B$ ne connaît pas l'origine des tampons.

- L'expérimentateur A filme alors systématiquement le comportement du bébé pendant $1 \mathrm{~min}$. La caméra super 8 étant sonore, les vocalisations ef les autres manifestations motrices sont enregistrées simultanément.

- L'expérimentateur $B$ tourne le dispositif de $180^{\circ}$ de façon à présenter à gauche le tampon qui était à droite et inversement; cela pour tenir comple des différences d'orientation latérale de la tête, d'un bébé à l'autre.

- L'expérimentateur A filme à nouveau cette situation pendant une minute.

FIG. 2. - Exemple de kinégramme permettant une étude quantifative des positions ef mouvements du nez et des deux bras du bébé dont le nez vient en contact avec un tampon de gaze imprégné des sécrétions du sein moternel (sécrétions lactées + sécrétions des glandes sudoripares ef sébacées) $(M)$ ef un tampon de gaze imprégné des mêmes sécrétions d'une autre mère (E). Les 2 tampons sont présentés alternativement à droite ef à gauche du bébé (demi-fest 1, puis demi-test 2), d̀ une minufe d'intervalle. Durée d'un demi-fest: $1 \mathrm{mn}$.

Les positions du nez et de chaque bras sont indiquées respectivement par des points, des éfoiles ef des croix, toutes les 36 images de films qui ont été pris à la vitesse de 18 images par sec. Ces positions ont été notées sur des feuilles de papier calque appliquées sur l'écran d'une visionneuse : elles sont ensuife reportées sur du papier quadrillé dont la surface des quadrats est de $2,5 \mathrm{~cm}^{2}$.

Dans cet exemple, l'étendue ef la densité des mouvements du nez sont données respectivement par 24 ef 3 quadrats, soient 60 et $7,5 \mathrm{~cm}^{2}$, puisque la surface d'un quadrat est de $2,5 \mathrm{~cm}^{2}$. Les mêmes calculs peuvent être faits pour l'étendue ef la densité des mouvements des deux bras.

(Kinégramme du test 4 . Anne-Claire, 3 jours).

- Positions du nez.

... Etendue des mouvements du nez.

* Positions du bras gauche

-. Etendue des mouvements du bras gauche.

+ Positions du bras droit.

_- Etendue des mouvements du bras droit. 


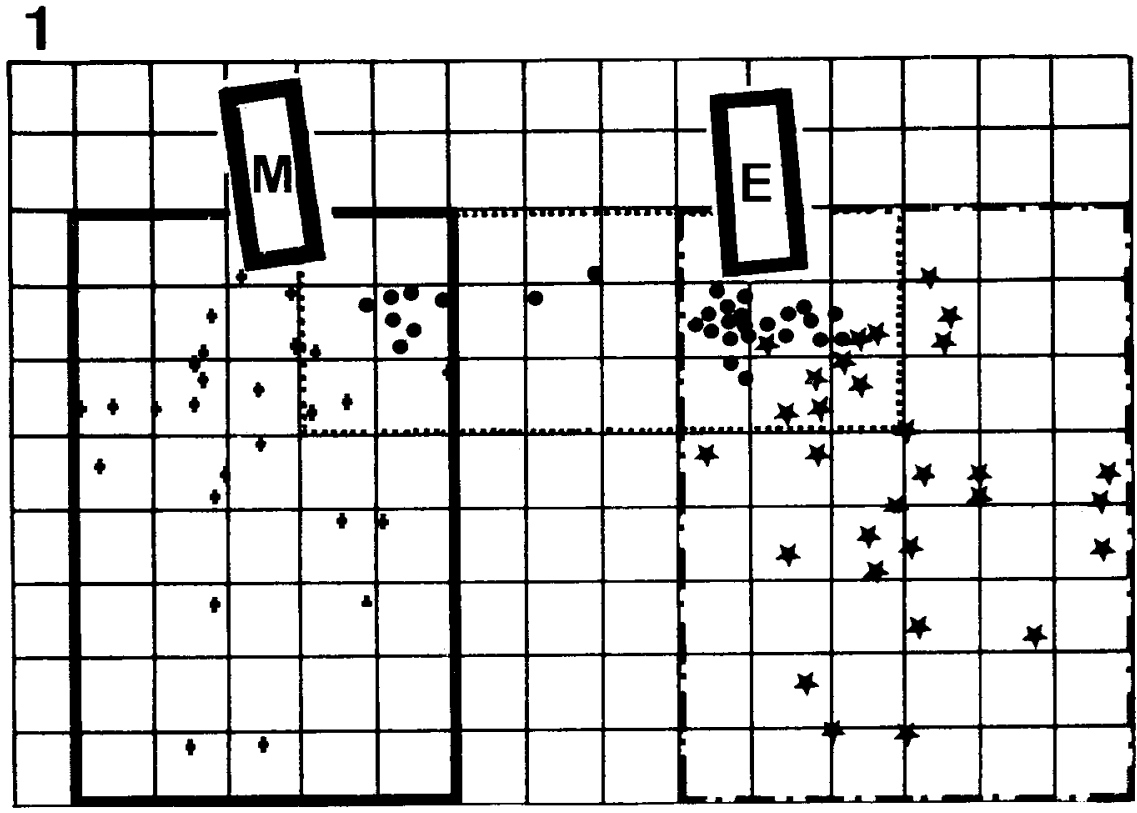

2

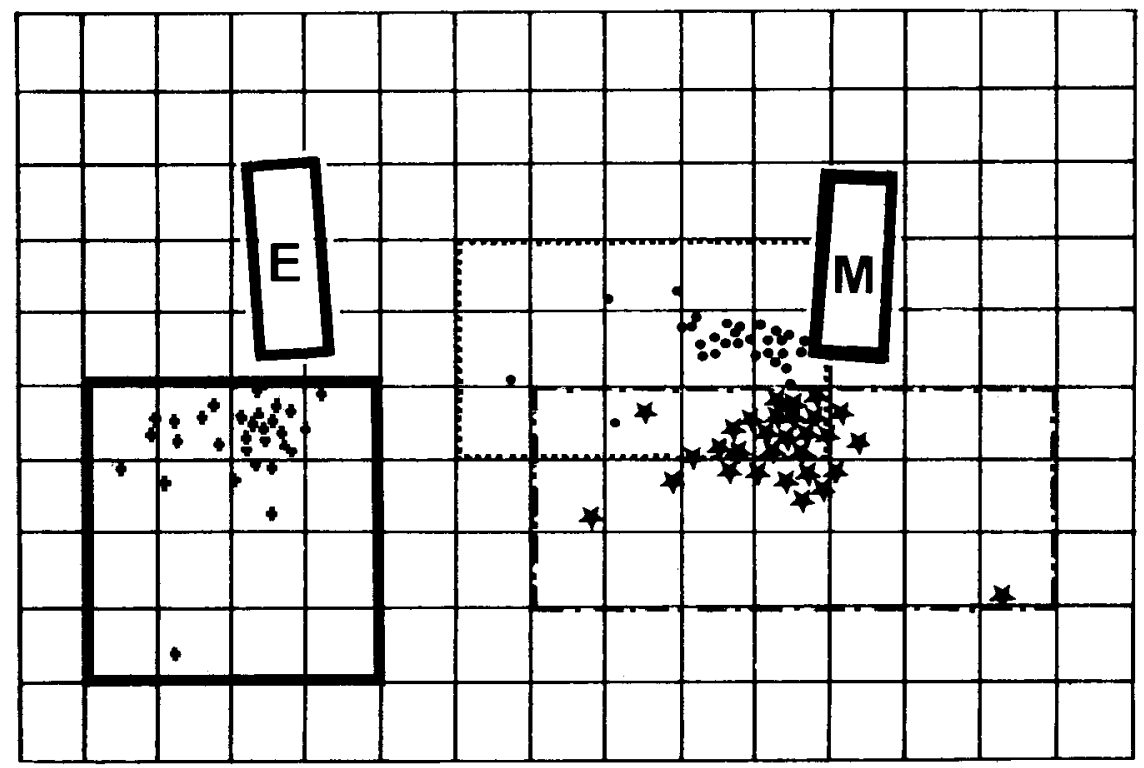

FIG. 2. 
Chaque test est donc analysé à partir de deux séquences de film de $1 \mathrm{~min}$ chacune.

Les tests sont effectués à la clinique les $2 \mathrm{e}, 4^{\mathrm{e}}, 6^{\mathrm{e}}, 8^{\mathrm{e}}, 10^{\mathrm{e}}$ et $12^{\mathrm{e}}$ jours après la naissance, puis à domicile aux âges de 1, 1,5 et 2 mois.

3) Analyse « kinégraphique » des réactions du bébé. - L'analyse des films se fait image par image au moyen d'une visionneuse : un papier calque est appliqué sur l'écran ef les positions du nez ef des deux membres supérieurs y sont notées toutes les 36 images (nous filmons à la viłesse de 18 images par sec.). On obtient ainsi 3 nuages de points pour chaque séquence de films (la moitié d'un test). fle papier calque est ensuite appliqué sur une grille dont chaque quadrat représente une surface réelle de $2,5 \mathrm{~cm}^{2}$ (fig. 2).

C'est à partir de chaque grille que nous avons extrait 2 paramètres qui permettent de caractériser la dynamique des mouvements du nez et des membres supérieurs :

a) l'étendue du mouvement est donnée par la surface totale des quadrats contenus dans l'enveloppe qui délimite toutes les positions du nez ou du bras, y compris les plus extrêmes ;

b) La densité des mouvements est donnée par la surface des quadrats adjacents dans lesquels s'inscrivent 50 p. 100 des positions du nez ou de chacun des bras.

C. - Tests de reconnaissance de l'odeur de l'enfant par la mère.

1) Recueil des odeurs de l'enfant. - On fait porter à chaque enfant une brassière à même la peau. Elle est recouverte par un tricoł pour qu'elle n'entre pas en contact avec la mère, lorsque celle-ci prend l'enfant dans ses bras. La brassière est portée de 24 à 48 h sans interruption. Tous les enfants ont les mêmes traitements hygiéniques (bains, application de crèmes sur le visage ef l'entrejambe, mais pas sur le tronc, zone que recouvre la brassière). Les tests sont effectués immédiatement après le recueil des brassières.

2) Déroulement des tests.

Conditions générales :

Ils sont faits tôt le matin, dans les chambres des mères :

- 3 à $4 \mathrm{~h}$ après la première tétée de la journée qui a lieu entre 5 et $6 \mathrm{~h}$, donc entre 8 et 10 h. Dans l'intervalle, il n'y a pas de contact entre la mère et l'enfant ;

- avant la foilette de la mère, sauf cas exceptionnel ;

- avant que la mère ne soit sollicitée par le personnel soignant ou le personnel administratif de la clinique.

- Les tests se font dans des chambres bien aérées, sans odeurs parasites décelables, dans une ambiance sonore et thermique constante.

- Les mères sont installées confortablement dans la situation qu'elles choisissent (lit, fauteuil).

Les tests.

On présente successivement à la mère 3 brassières dont celle de son bébé. L'ordre de présentation et la position de la brassière de celui-ci par rapport aux autres sont effectués au hasard. On répète cette opération 5 fois en changeant ou non les brassières de référence (au total 7 à 9 brassières sont utilisées une ou plusieurs fois, de façon aléatoire). 
Les choix sont ainsi notés :

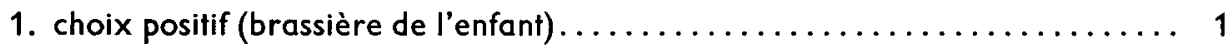

2. choix négatif (brassière d'un autre enfant) $\ldots \ldots \ldots \ldots \ldots \ldots \ldots \ldots \ldots \ldots$

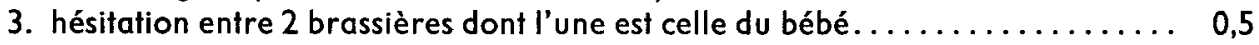

4. hésitation entre 2 brassières portées par 2 enfants étrangers.......... 0

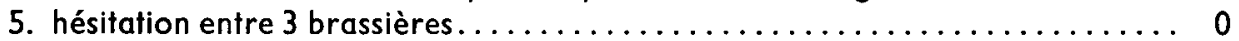

Nous effectuons 2 types de tests.

- Tests directifs. - Les yeux de la mère sont soigneusement bandés. 3 brassières sont appliquées successivement sous le nez de la mère par un expérimentateur $A$ qui ne connaît pas leur appartenance. La zone présentée est celle qui a été en contact avec le dessous des bras, les côtés et le dos des bébés. Un expérimentateur B note les choix de la mère sans aucun commentaire et sans réaction particulière. Ces tests sont répétés 5 fois chaque jour pendant 10 ou 12 jours.

- Tests de libre-choix. - Pour éviter toute interaction avec les 2 expérimentateurs, même lorsque ceux-ci opèrent en aveugle, le protocole suivant a été aussi utilisé : nous posons directement devant la mère un lot de 3 brassières dont celle de son bébé. Les yeux soigneusement bandés et les mains gantées, la mère prend le temps qu'elle désire pour explorer olfactivement chaque brassière. Un expérimentateur note les choix de la mère, à distance, sans aucun commentaire ni réaction particulière. Ces tests sont répétés 5 fois tous les 2 jours.

\section{2. - La discrimination des odeurs spécifiques par l'enfant de 3 à 5 ans.}

Jusqu'à ce jour, nous nous sommes limités à l'étude de la discrimination de l'odeur maternelle par l'enfant.

Nous avons demandé à des mères d'enfants fréquentant l'école maternelle de porter un tee-shirt blanc à même la peau, par-dessus le soutien-gorge pour ne pas mélanger des sécrétions lactées éventuelles aux sécrétions des glandes sudoripares et sébacées. Tous les tee-shirts sont identiques; la durée du port du tee-shirt est de 2 à 3 jours. Les mères ont pour consigne de ne pas changer leurs habitudes : elles conservent l'utilisation de leurs eaux de toilette, savons, parfums ; ou bien elles n'en utilisent pas, si elles ne le font pas habituellement.

Chaque enfant est soumis à plusieurs expériences de choix entre le tee-shirt maternel ef celui d'une autre mère : celui-ci change ou reste le même, selon un protocole aléatoire ; parfois, l'enfant est soumis à un choix entre deux tee-shirts de mères étrangères. Chaque expérience comporte un double choix : entre le tee-shirt maternel, présenté en premier, et le tee-shirt de l'autre mère, puis entre celui-ci et le tee-shirt maternel ; ou inversement, d'une façon aléatoire. On peut ainsi soumettre les enfants de 3 à 7 doubles choix par matinée, selon la disponibilité de chacun et selon les jours. Les expériences se déroulent dans la salle de jeux de l'école maternelle ou dans la classe, alors que les autres enfants sont respectivement dans la classe ou la salle de jeux. L'instifutrice fait sentir successivement à l'enfant les 2 tee-shirts que lui a remis l'expérimentateụr, puis 2 autres ou l'un de ces tee-shirts et un autre, selon le protocole établi par l'expérimentateur. Elle demande à l'enfant : « lequel tu prends », « lequel tu choisis », «lequel tu aimes ». L'expérimentateur note les réponses verbales et comportementales de l'enfant et de l'institutrice. 
Nous avons fait la somme des choix positifs (choix du tee-shirt maternel) par rapport à l'ensemble des choix proposés à l'enfant. Le plus souvent, tous les enfants des échantillonnages ont été testés les mêmes jours, mais il est arrivé que certains aient été absents certains jours et aient été testés le lendemain, ou n'aient pas été testés certaines semaines.

\section{Résultats.}

A. - Reconnaissance de l'odeur de la mère par des bébés de 3 à 10 jours.

La figure 3 permet de comparer l'étendue et la densité des mouvements du nez ef des 2 bras de 10 enfants * de 3 à 10 jours auxquels on a présenté successivement l'odeur du sein majernel et l'odeur d'un tampon témoin «olfactivement neutre ».
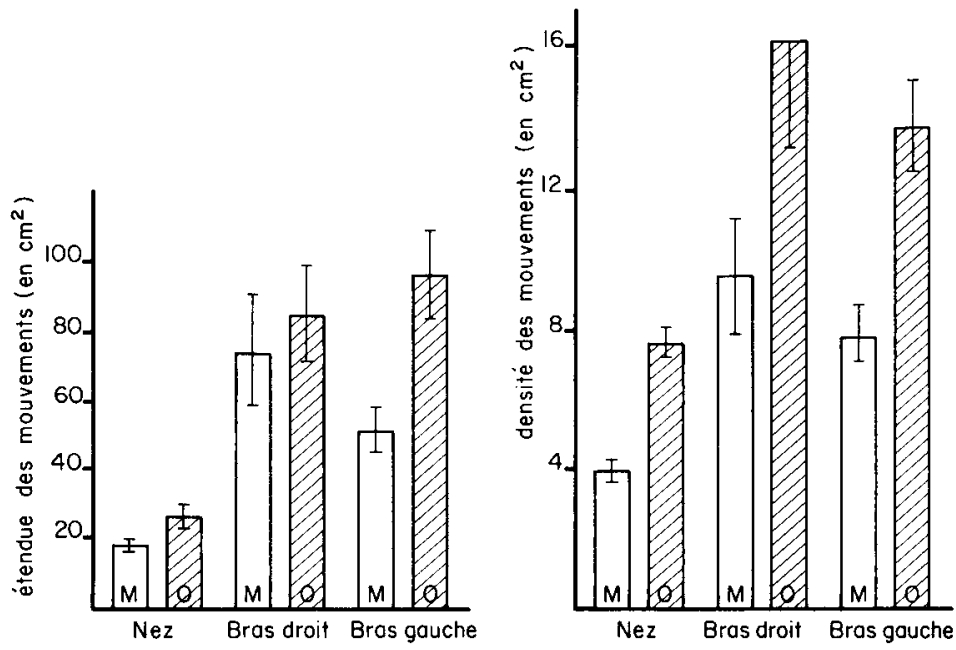

FIG. 3. - Etendue ef densité des mouvements du nez (et donc de la tête) et des deux bras de 10 bébés de 3 à 10 jours auxquels on a présenfé successivement à une minute d'intervalle un tompon imprégné de l'odeur du sein mafenel $(M)$ ef un lampon olfactivement « neutre " (0). Chaque moyenne est représentée avec son erreur standard.

La figure 4 permet de comparer ces mêmes paramètres chez 8 enfants de 3 à 10 jours auxquels on a présenté successivement l'odeur du sein maternel et l'odeur du sein d'une autre mère.

Dans les 2 cas, le contact du nez du nouveau-né avec la source d'odeurs maternelles se traduit de façon significative par une diminution de l'étendue ef de la densité des mouvements du nez (et donc de la tête) et des 2 bras, sauf pour l'étendue des mouvements du bras droit dans la figure 3 (tous les enfants testés sont nourris au sein). article.

* L'analyse des films réalisés sur les autres enfants n'a pu être achevée avant la remise du présent 

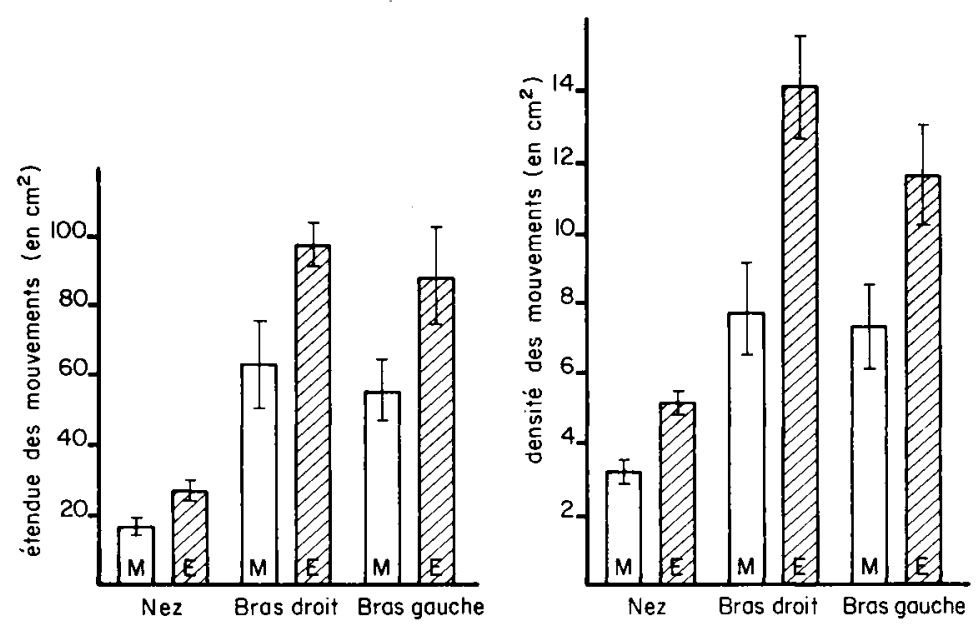

FIG. 4. - Etendue ef densité des mouvements du nez (et donc de la tête) et des deux bras de 8 bébés de 3 à 10 jours auxquels on a présenté successivement à une minute d'intervalle un tampon imprégné de l'odeur du sein maternel $(\mathrm{M})$ et un fampon imprégné de l'odeur du sein d'une mère "éfrangère " $(\mathrm{E})$. Chaque moyenne est représentée avec son erreur standard.

B. - Reconnaissance de l'odeur du bébé par la mère.

1. - Mise en évidence ef évolution au cours des 10 premiers jours.

L'ensemble des 20 mères testées du $2^{e}$ au $10^{e}$ jour après la naissance $d u$ bébé reconnaissent de façon significative l'odeur véhiculée par la brassière de leur enfant par rapport à celle des brassières portées par d'autres enfants : au total 59,70 p. 100 de choix de la brassière portée par leur enfant : le test $d u \chi^{2}$ donne une différence significative par rapport au hasard $(P<0,01)$.

La figure 5 montre comment évoluent du $2^{\mathrm{e}}$ au $10^{\mathrm{e}}$ jour les pourcentages de choix des mères auxquelles on a présenté au hasard 3 brassières portées par 3 enfants différents dont celui de la mère.

FIG. 5. - Evolution du pourcentage de choix de l'odeur corporelle du bébé par la mère du $2^{e}$ au $10^{\mathrm{e}}$ jour post-natal (tous résultats confondus : tous les jours chez toutes les 20 mères).

- - : choix de la brassière portée par l'enfant de la mère ;

-—o : choix de la brassière portée par un bébé étranger :

---- : hésitations entre deux brassières, dont celle de l'enfant de la mère. Chaque moyenne est donnée avec son erreur standard.

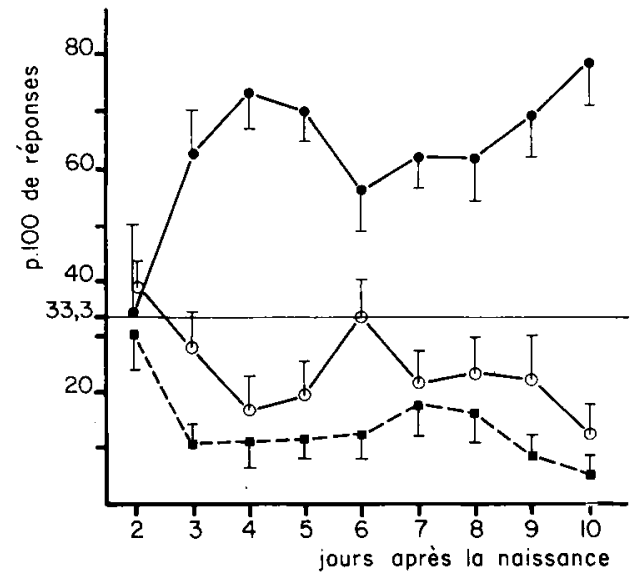


Au $2^{\mathrm{e}}$ jour post-natal, le choix des 20 mères paraît se faire au hasard. Mais dès le $3^{e}$ jour, les mères choisissent de façon significative la brassière de leur propre enfant, ce qui s'accompagne non seulement d'une chute des choix en faveur de la brassière d'un autre enfant, mais aussi d'une chute des hésitations entre la brassière de leur enfant et celle d'un enfant « étranger ». Puis, à partir du $5^{\mathrm{e}}$ jour, on observe une diminution dans la proportion de choix en faveur de la brassière de l'enfant. Cette proportion augmente de nouveau du $8^{\mathrm{e}}$ au $10^{\mathrm{e}}$ jour où elle atteint 80 p. 100.

Cependant, les pourcentages moyens de choix de la brassière portée par l'enfant peuvent varier de 30 à 94 p. 100 d'une mère à l'autre (tabl. 1). Nous avons donc commencé à rechercher les facteurs qui pouvaient être à l'origine des différences de choix entre les mères.

\section{TABLEAU 1}

Hétérogénéité des pourcentages moyens de choix de la brassière de l'enfant par la mère

\begin{tabular}{lc}
\hline Choix de la brassière de l'enfant & Nombre de mères \\
\hline supérieur à 80 p. $100 \ldots \ldots \ldots \ldots \ldots$ & 3 \\
\hline de 60 à 80 p. $100 \ldots \ldots \ldots \ldots \ldots \ldots \ldots$ & 6 \\
\hline de 50 à 59 p. $100 \ldots \ldots \ldots \ldots \ldots \ldots \ldots \ldots$ & 6 \\
\hline inférieur à 50 p. $100 \ldots \ldots \ldots \ldots \ldots \ldots \ldots$
\end{tabular}

\section{2. - Facteurs affectant la reconnaissance olfactive de l'enfant par sa mère.}

Nous avons étudié l'influence de 4 paramètres sur la variabilité de la reconnaissance olfactive de l'enfant par la mère : le sexe de l'enfant, la parité de la mère, le mode d'alimentation de l'enfant et les modalités de contact entre la mère et l'enfant à la naissance de l'enfant.

1) Le sexe de l'enfant. - Ce paramètre ne semble pas affecter la reconnaissance de l'odeur de l'enfant (62,57 p. 100 de réponses positives pour les mères de filles, 60,44 p. 100 pour celles de garçons).

2) La parité de la mère. - La comparaison des pourcentages $(65,09$ p. 100 chez les mères primipares ; 56,9 p. 100 chez les mères multipares) fait ressortir un pourcentage de choix de l'odeur de l'enfant plus élevé chez les mères primipares que chez les mères multipares. Mais le petit nombre de données actuellement disponibles n'autorise pas de comparaison statistique utile.

3) Le mode d'allmentation du nouveau-né. - Sur les 20 mères suivies, 15 nourrissaient leur enfant au sein et 5 au biberon. Le pourcentage moyen de choix de la brassière imprégnée de l'odeur de l'enfant par la mère est de 63,96 p. 100 chez les mères allaitantes et de 53,53 p. 100 chez les mères qui donnent le biberon ; mais, là aussi, le petit nombre de données ne peut déboucher sur une étude statistique utile. 
4) Les modalités du contact précoce entre la mère ef l'enfant. - Ce facteur est incontestablement celui qui influence le plus les pourcentages de choix de la brassière de l'enfant par la mère. Trois types de situations ont été considérées (fig. 6 ) :

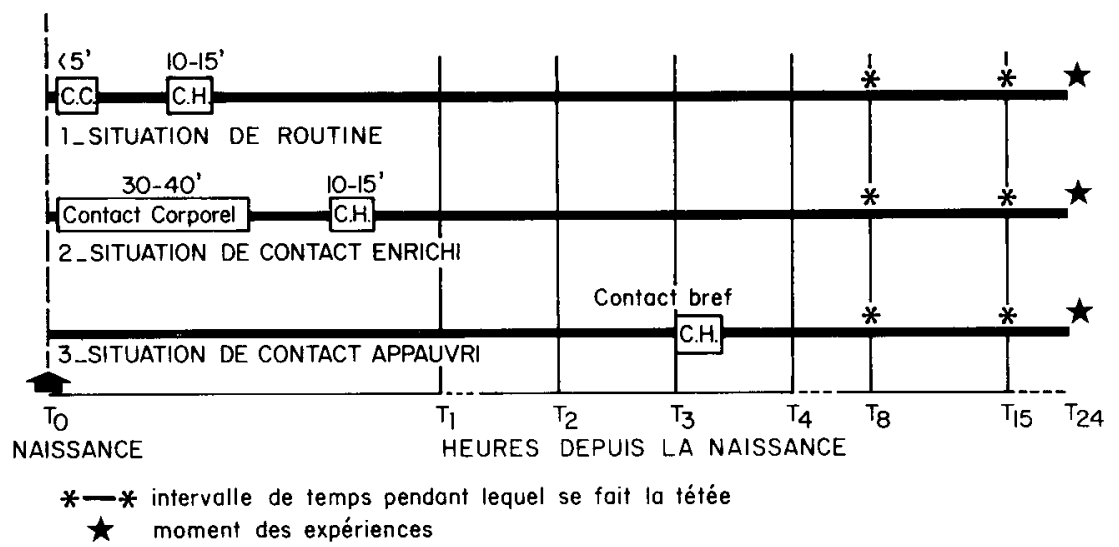

FIG. 6. - Les trois situations relationnelles entre la mère ef son bébé, de la naissance aux premiers tests de reconnaissance de la brassière du bébé par la mère.

C.C. : Contact corporel entre la mère et l'enfant ; C.H. : Contact entre la mère et l'enfant habillé. Les expériences ont lieu tous les jours au même moment. $T_{0} \grave{a} T_{24}$ : heures depuis la naissance.

a) Situation de routine : la mère et son enfant ont à la naissance un contact corporel CC dit de routine : l'enfant est déposé nu sur le ventre maternel pendant une durée inférieure à $5 \mathrm{~min}$. ; après avoir été pesé, lavé ef habillé, il est remis en contact avec sa mère pendant 10 à $15 \mathrm{~min}(\mathrm{CH})$. Cette situation a été vécue par 8 couples mère-enfant.

b) Situation de contact enrichi : la mère et l'enfant restent plus longtemps « peau à peau » (contact corporel). Dès la naissance, le nouveau-né est déposé nu sur le ventre maternel et y reste pendant 30 à $40 \mathrm{~min}$. Au cours de ce contact peau à peau, nous favorisons la mise au sein de l'enfant. Après avoir été pesé, lavé ef habillé, l'enfant est remis à la mère pendant 10 à $15 \mathrm{~min}(\mathrm{CH})$. Cette situation a été vécue par 7 couples mère-enfant.

c) Situation de contact appaurri : la mère ef l'enfant ne sont pas mis en contact immédiatement après la naissance. C'est le cas de 5 mères qui ont subi une intervention chirurgicale au cours de l'accouchement (césariennes, délivrances artificielles...). Ces mères ne voient leur enfant que $3 \mathrm{~h}$ en moyenne après la naissance (la plupart n'ont accepté qu'un contact bref avec l'enfant habillé $(\mathrm{CH})$ au moment de la présentation).

Le tableau 2 montre que, pour l'ensemble des 10 jours après la naissance, seules toutes les mères qui ont eu un contact enrichi avec leur enfant ont un pourcentage de choix positifs supérieur à 60 p. 100. La figure 7 indique que, dans ce cas, 60 p. 100 de choix positifs sont atteints au $2^{e}$ jour post-natal. Alors que, le $2^{e}$ jour, il y a une différence significative entre les mères ayant eu une situation de contact enrichi ef celles qui ont eu une situation de routine, il n'y en a plus du $3^{e}$ au $6^{6}$ jour. En revanche, il y a une différence significative entre ces 2 populations de mères et celles qui ont eu un 
TABLEAU 2

Pourcentages moyens de choix maternels de la brassière du bébé $d u 2^{\theta}$ au $10^{\mathrm{e}}$ jour après la naissance (tous résultats confondus)

\begin{tabular}{|c|c|c|c|c|}
\hline $\begin{array}{l}\text { Réponses } \\
\text { positives } \\
\text { (p. 100) }\end{array}$ & $\begin{array}{c}\text { Réponses } \\
\text { négatives } \\
\text { (p. 100) }\end{array}$ & $\begin{array}{l}\text { Réponses } \\
\text { hésitantes } \\
\text { (p. 100) }\end{array}$ & $\begin{array}{l}\text { Nombre } \\
\text { de tests }\end{array}$ & $\begin{array}{l}\text { Type de relation } \\
\text { entre mère et enfant }\end{array}$ \\
\hline $\begin{array}{l}94,12 \\
84 \\
80,79 \\
76,94 \\
71,80 \\
71,44 \\
67,65 \\
61,76 \\
60,18 \\
58,82 \\
58,82 \\
57,78 \\
56,82 \\
56 \\
52,78 \\
42,86 \\
41,17 \\
41,02 \\
30 \\
29,42\end{array}$ & $\begin{array}{c}0 \\
2 \\
11,56 \\
5,09 \\
15,38 \\
14,28 \\
32,35 \\
35,29 \\
18,37 \\
0 \\
35,29 \\
33,33 \\
38,64 \\
16 \\
38,89 \\
45,71 \\
58,83 \\
23,08 \\
30 \\
17,64\end{array}$ & $\begin{array}{c}5,88 \\
14 \\
7,63 \\
17,96 \\
12,82 \\
14,28 \\
0 \\
2,95 \\
22,43 \\
41,18 \\
5,89 \\
8,89 \\
4,54 \\
28 \\
8,33 \\
11,43 \\
0 \\
35,90 \\
40 \\
52,94\end{array}$ & $\begin{array}{l}18 \\
65 \\
26 \\
39 \\
43 \\
67 \\
51 \\
34 \\
93 \\
17 \\
17 \\
89 \\
44 \\
50 \\
36 \\
43 \\
44 \\
39 \\
30 \\
34\end{array}$ & $\begin{array}{l}\text { Contact }+ \\
\text { Contact }+ \\
\text { Contact }+ \\
\text { Contact }+ \\
\text { Contact + } \\
\text { Contact }+ \\
\text { Contact R } \\
\text { Contact R } \\
\text { Contact + } \\
\text { Contact R } \\
\text { Contact R } \\
\text { Contact - } \\
\text { Contact R } \\
\text { Contact - } \\
\text { Contact R } \\
\text { Contact R } \\
\text { Contact - } \\
\text { Contact - } \\
\text { Contact - } \\
\text { Contact R }\end{array}$ \\
\hline
\end{tabular}

Réponses positives : choix de la brassière portée par son bébé. Réponses négatives : choix de la brassière portée par un autre bébé. Contact $R$ : situation de routine (voir fig. 6 ). Contact + : sifuation de contact enrichi. Contact - : situation de contact appauvri.

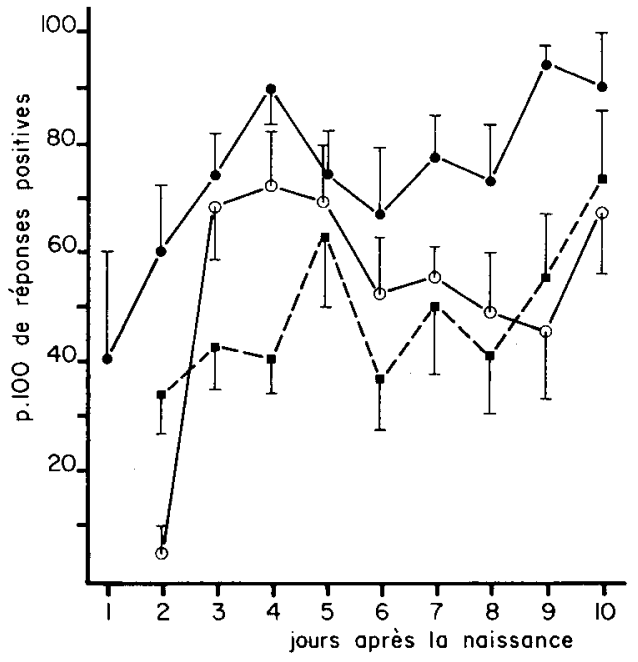

FIG. 7. - Evolution du pourcentage de choix de l'odeur corporelle du bébé par la mère du $2^{\mathrm{e}}$ au $10 \mathrm{e}$ jour post-natal, en fonction du mode de relation entre la mère et l'enfant depuis la naissance.

-- : le couple mère-enfant est dans la situation de contact enrichi ( $n=7)$; 0 - le couple mère-enfant est dans la situation de routine $(n=8)$; $-\cdots--$ : le couple mère-enfant est dans la situation de contact appaurri $(n=5)$.

Réponses positives : choix de la brassière de l'enfant par la mère de celui-ci.

Chaque moyenne est donnée avec son erreur standard. 
contact appaurri du $3^{\mathrm{e}}$ au $6^{\mathrm{e}}$ jour (à l'exception du $5^{\mathrm{e}}$ jour, pour les mères qui ont eu la situation de routine).

Au 7 e jour, le pourcentage de choix des mères au contact enrichi s'accroît de nouveau ef devient significativement plus élevé que celui des autres mères jusqu'au $9 \mathrm{e}$ jour. C'est aussi à partir du $7 \mathrm{e}$ jour que les courbes des mères au contact de routine et des mères au contact appauvri tendent à évoluer de la même façon.

C. - Reconnaissance de l'odeur corporelle « globale » de la mère par l'enfant de 3 à 5 ans.

Le tableau 3 montre que le $1 / 3$ des enfants de 2 populations de 26 enfants de 45 à 58 mois, étudiées dans 2 classes par 2 chercheurs différents, ont choisi de façon signifi-

TABLEAU 3

Etude quantitative du choix de tee-shirts imprégnés de l'odeur maternelle par rapport à des tee-shirts imprégnés de l'odeur d'une autre mère, par 26 enfants de 45 à 58 mois

\begin{tabular}{|c|c|c|c|c|c|c|}
\hline \multirow[b]{2}{*}{$\begin{array}{l}\text { Nombre } \\
\text { d'expé- } \\
\text { riences }\end{array}$} & \multirow[b]{2}{*}{$x_{1}$} & \multirow[b]{2}{*}{$x_{2}$} & \multirow[b]{2}{*}{$\begin{array}{l}\text { Comparaison au } \\
\text { moyen de la loi } \\
\text { binomiale (Siegel) }\end{array}$} & \multirow[b]{2}{*}{$\begin{array}{l}\text { Pourcentage } \\
\text { de choix du } \\
\text { tricot } \\
\text { maternel } \\
\text { (p. 100) }\end{array}$} & \multicolumn{2}{|c|}{ Pourcentage d'enfants } \\
\hline & & & & & $\begin{array}{l}\text { qui choisissent } \\
\text { de façon } \\
\text { signi- } \\
\text { ficative }\end{array}$ & $\begin{array}{c}\text { le tricot } \\
\text { maternel } \\
\text { de façon non } \\
\text { significative } \\
\text { mais dans plus } \\
\text { de } 60 \text { p. } 100 \\
\text { des choix }\end{array}$ \\
\hline
\end{tabular}

\begin{tabular}{|c|c|c|c|c|c|c|c|}
\hline $\begin{array}{l}\text { Julien ..... } \\
\text { Stéphanie ... } \\
\text { Gilda ...... } \\
\text { François ... } \\
\text { Laurence... } \\
\text { Luc ...... } \\
\text { Claudine ... } \\
\text { Jean-Philippe }\end{array}$ & $\begin{array}{l}28 \\
37 \\
37 \\
35 \\
27 \\
23 \\
24 \\
21\end{array}$ & $\begin{array}{l}22 \\
26 \\
26 \\
24 \\
19 \\
17 \\
17 \\
15\end{array}$ & $\begin{array}{r}6 \\
11 \\
11 \\
11 \\
8 \\
6 \\
7 \\
6\end{array}$ & $\begin{array}{l}\text { D.S. } \mathrm{p}=0.01 \\
\text { D.S. } \mathrm{p}=0.0107 \\
\text { D.S. } \mathrm{p}=0.0107 \\
\text { D.S. } \mathrm{p}=0.022 \\
\text { D.S. } \mathrm{p}=0.027 \\
\text { D.S. } \mathrm{p}=0.030 \\
\text { D.S. } \mathrm{p}=0.034 \\
\text { D.S. } \mathrm{p}=0.039\end{array}$ & $\begin{array}{l}78 \\
73 \\
70 \\
68 \\
70 \\
73 \\
70 \\
71\end{array}$ & 30 p. 100 & \\
\hline $\begin{array}{l}\text { Stéphanie ... } \\
\text { Damien ... } \\
\text { Julien ..... } \\
\text { Claudie ... } \\
\text { Sophie .... } \\
\text { Valérie .... } \\
\text { Anne ..... } \\
\text { Isabelle ... } \\
\text { Lydie ..... } \\
\text { Sandrine ... }\end{array}$ & $\begin{array}{l}15 \\
36 \\
31 \\
28 \\
23 \\
25 \\
28 \\
21 \\
21 \\
20\end{array}$ & $\begin{array}{l}11 \\
23 \\
20 \\
18 \\
15 \\
16 \\
17 \\
13 \\
13 \\
12\end{array}$ & $\begin{array}{r}4 \\
13 \\
11 \\
10 \\
8 \\
9 \\
11 \\
8 \\
8 \\
8\end{array}$ & $\begin{array}{l}\text { D.N.S. } p=0.059 \\
\text { D.N.S. } p=0.067 \\
\text { D.N.S. } p=0.075 \\
\text { D.N.S. } p=0.093 \\
\text { D.N.S. } p=0.105 \\
\text { D.N.S. } p=0.115 \\
\text { D.N.S. } p=0.171 \\
\text { D.N.S. } p=0.192 \\
\text { D.N.S. } p=0.192 \\
\text { D.N.S. } p=0.25\end{array}$ & $\begin{array}{l}73 \\
63 \\
64 \\
64 \\
65 \\
64 \\
60 \\
61 \\
61 \\
60\end{array}$ & & 39 p. 100 \\
\hline $\begin{array}{l}\text { Sullivan } \ldots . \\
\text { Sophie .... } \\
\text { Magalie ... } \\
\text { Sylvain .... } \\
\text { Sophie .... } \\
\text { Christophe . } \\
\text { Bénédicte .. } \\
\text { Sonia ..... }\end{array}$ & $\begin{array}{c}16 \\
32 \\
30 \\
36 \\
17 \\
28 \\
\text { Refus } \\
\text { Refus }\end{array}$ & $\begin{array}{r}9 \\
17 \\
16 \\
18 \\
8 \\
12 \\
\text { de } \\
\text { de }\end{array}$ & $\begin{array}{r}7 \\
15 \\
14 \\
18 \\
9 \\
16\end{array}$ & $\begin{array}{l}\text { D.N.S. } P=0.402 \\
\text { D.N.S. } P=0.456 \\
\text { D.N.S. } P=0.468 \\
\text { D.N.S. } \\
\text { D.N.S. } \\
\text { D.N.S. } \\
\text { choisir } \\
\text { choisir }\end{array}$ & $\begin{array}{l}56 \\
53 \\
53 \\
50 \\
47 \\
48\end{array}$ & & \\
\hline
\end{tabular}

$x_{1}=$ Nombre de choix du tee-shirt maternel.

$x_{2}=$ Nombre de choix du tee-shirt d'une autre mère. 
cative le tee-shirt maternel. Plus du 1/3 des autres l'ont choisi dans plus de 60 p. 100 des cas.

Ces résultałs ne peuvent s'expliquer que par la reconnaissance de l'odeur du tee-shirt maternel par au moins $1 / 3$ des enfants.

Il faut maintenant comprendre pourquoi certains enfants reconnaissent et choisissent le tee-shirt maternel, pourquoi d'autres fluctuent dans leurs choix d'une série d'expériences à l'autre, pourquoi d'autres ne choisissent pas le tee-shirt maternel alors qu'ils le reconnaissent peut-être, pourquoi d'autres enfin choisissent au hasard. II faut aussi étudier les causes des modifications odorantes des tee-shirts maternels, au moins de certains, d'une série d'expériences à l'autre : état physiologique de la mère, changement d'eau de toilette, etc...

\section{Conclusions et discussion.}

Le jeune enfant paraît donc reconnaître très tôt, au moins à partir du $4 \mathrm{e}$ jour, le complexe odorant constitué par les sécrétions lactées ef les sécrétions sébacées et sudoripares du mamelon de sa mère. Nous confirmons ainsi les observations de Mac Farlane (1975), mais avec des critères et une méthode de quantification qui reposent sur l'activité motrice de la tête ef des membres supérieurs et non sur la seule orientation de la tête par rapport à la source odorante. Une étude comparative avec des prématurés doit nous permettre de comprendre encore plus finement la genèse de la discrimination olfactive du nouveau-né.

Les premiers liens entre la mère et son enfant s'établissent donc entres autres, sur des bases olfactives. On retrouve ainsi un mécanisme d'attachement « mère-jeune» très répandu chez les Mammifères dits « macrosmates». Or, on sait qu'il existe des relations anatomo-fonctionnelles particulièrement étroites entre les cellules sensorielles de la muqueuse olfactive ef le système limbique, dont le rôle est si important dans les comportements relationnels des Mammifères (Karli, 1968, 1976). On peut donc se demander si une absence ou une perturbation des relations olfactives entre le nouveau-né et sa mère n'entraînent pas, au moins si elles sont durables, des perturbations dans le développement affectif de l'enfant.

Il apparaît aussi que la mère reconnaît l'odeur de son enfant, au moins lorsqu'elle a eu un contact corporel avec lui à la naissance. La discrimination olfactive de la mère est d'autant plus marquée que la durée du contact avec l'enfant a été plus longue. L'étude de l'évolution de cette discrimination olfactive au cours des 10 jours qui suivent la naissance de l'enfant fait ressortir 3 particularités : 1. - Une diminution entre le $5^{\mathrm{e}}$ et le $6^{\mathrm{e}}$ jour chez les mères qui ont eu un contact corporel de routine ( $<5$ minutes) ou un contact corporel approfondi (de 30 à $40 \mathrm{~min}$ ) avec leur enfant à la naissance. Si cela se confirme (la différence n'est pas statistiquement significative entre la valeur moyenne du $4 \mathrm{e}$ jour et la valeur moyenne du 6e jour, mais le phénomène apparaît chez toutes les mères), deux types d'hypothèses peuvent être faites : a) l'odeur du nouveau-né pourrait changer (par exemple : d'une odeur « amniotique », il pourrait passer à une odeur plus «personnelle », faite de sécrétions des glandes sébacées et sudoripares, que la mère devrait apprendre à reconnaître); b) des modifications 
physiologiques post-partum particulières de la mère pourraient influencer ses capacités de discrimination olfactive. Nous examinons actuellement ces 2 types d'hypothèses.

- A partir du $6^{e}$ jour, les mères qui ont eu un contact approfondi avec leur nouveau-né à la naissance reconnaissent mieux l'odeur de celui-ci que les autres mères. La qualité sensorielle des relations entre la mère et son enfant à la naissance jouerait donc un rôle facilitateur dans la reconnaissance olfactive de celui-ci par la première après la courte période de « dépression » du $4^{\mathrm{e}}$ au $6^{\mathrm{e}}$ jour.

- Cependant, à partir du 9e jour, la reconnaissance olfactive du nouveau-né paraît augmenter brusquement chez les mères qui n'ont eu qu'un contact de routine avec l'enfant juste après la naissance et chez celles qui n'ont eu avec lui qu'un contact bref, $3 \mathrm{~h}$ après la naissance. Les recherches en cours nous permettront de savoir si cette évolution se confirme ou s'accentue après le 10e jour, par rapport aux mères qui ont eu un contact approfondi avec leur enfant à la naissance.

La méthode de quantification que nous avons utilisée pour étudier le comportement du bébé à deux stimulations olfactives différentes peut être utilisée pour étudier la discrimination éventuelle que fait le bébé entre deux sons ou vocalisations spécifiques (émis par la mère, mais aussi par le père ou d'autres personnes) ; ou bien entre la perception éventuelle à distance des formes par rapport aux mouvements, à des âges précis, alors que la genèse de ces discriminations n'est pas encore très bien établie. Cette méthode peut aussi être utilisée pour cerner le caractère attractif ou aversif des laits artificiels et des drogues, parfois nécessaires pour traiter certaines affections et maladies du bébé. De façon générale, elle peut permettre d'isoler avec précision les facteurs d'environnement qui ont un caractère attractif, aversif, neutre, etc... pour le bébé. C'est ce que nous avons entrepris.

L'étude simultanée des différentes odeurs corporelles, des bruits du cœur, des intonations de voix (de la mère), des vocalisations (du nouveau-né), des confacts corporels, des stimulations labyrinthiques, des mimiques et autres configurations visuelles spécifiques, etc... nous permetta peut-être de comprendre encore plus finement le rôle respectif des différents types de stimulations sensorielles à partir desquelles se construisent, se renforcent ou se détériorent les échanges entre la mère et le bébé, à telle ou telle période post-natale.

Par ailleurs, une partie des enfants de 3 à 5 ans reconnaît aussi de façon significative l'odeur maternelle. Les bases olfactives de l'attachement entre la mère ef son enfant subsistent donc longtemps, au moins dans le $1 / 3$ des cas. II faut maintenant étudier les causes de la déficience ou de l'absence de discrimination olfactive spécifique chez le jeune enfant et voir si cela s'accompagne d'une genèse particulière de ses systèmes relationnels. II faut aussi analyser l'influence éventuelle de l'état physiologique de la mère ef de l'enfant sur les fluctuations de la discrimination olfactive de celui-ci. 


\section{Références}

ENGEN T., LIPSITT L. P., KAY H., 1963. Olfactory responses and adaptation in human neonate. J. comp. Physiol. Psychol., 56, 73-77.

HOLD B., SCHLEIDT M., 1977. The importance of human odour in non-verbal communication. Zeitschr Tierpsychol., 43, 225-238.

HUTT S. J., LENARD H., PRECHTL H. F. R., 1969. Psychophysiological studies in newborn infants, 127-172. In REESE H. W., LIPSITT L. P., Advances in child development and behaviour. Acad. Press, London, New York.

KARLI P., 1968. Système limbique ef processus de motivation. J. Physiol., 60, Suppl. 1, 3-148.

KARLI P., 1976. Rhinencéphale et comportements socio-affectifs, 149-163. In Rhinencéphale, neurotronsmetteurs ef psychoses, Symp. Bel Air, V, Genève.

Mac FARLANE A., 1975. Olfaction in the development of social preferences in the human neonate, 103-117. In Parent-infant inter-action. Ciba Found. Symp., 33.

MONTAGNER H., 1974a. Communication non verbale et discrimination olfactive chez les jeunes enfants : approche éthologique. In L'unité de l'Homme, 246-270. Edit. du Seuil, Paris.

MONTAGNER H., ARNAUD M., JEANDROZ M., RENNER N., ROSIER M., HENRY E., HENRY J. C., HERBSTMEYER M., HUMBERT Y., KARSENTY Ch., CHAVANNE J., 1974b. Les activités ludiques du jeune enfant : jeu ou ontogenèse? Vers l'Education nouvelle, $n^{\circ}$ hors-série, 15-44.

MONTAGNER H., 1978. L'Enfant et la communication. Stock, Paris.

PRECHTL H. F. R., 1965. Problems of behavioral studies in the newborn infant. In LEHRMAN D. S., HINDE, R. A., SHAW E., Adv. Study Behav., 1, 75-98. Acad. Press, London, N. Y.

SCHAAL B., HERTLING E., MONTAGNER H., 1979. Mutual olfactory recognition between the human mother and her newborn infant. Ethol. Sociobiol. (à paraître).

STEINER J. E., 1973. The gustofacial response : observation on normal and anencephalic newborn infants. In BOSMA J. F. 4th Symp. Oral sensation and perception (DHEW Publ. No 73-546) Dept Health, Education and Welfare, Bethesda, Md, U.S.A. 